\title{
Avaliação dos Critérios de Elegibilidade do Programa Territórios da Cidadania ${ }^{1}$
}

\author{
Pedro Gomes Andrade², Julia Celia Mercedes Strauch ${ }^{3}$ \\ e Gustavo Ferreira ${ }^{4}$
}

Resumo: O Programa Territórios da Cidadania (PTC) é uma das mais relevantes políticas de desenvolvimento territorial vigentes no Brasil, principalmente no que tange sua abrangência e importância. Trata-se de uma política pública social que apresenta escopo diferenciado, com enfoque territorial, gestão social e interministerial. Apesar de o PTC ser oriundo de um processo evolutivo de políticas públicas como: Programa Nacional de Fortalecimento da Agricultura Familiar (Pronaf) e Programa de Desenvolvimento Sustentável dos Territórios Rurais (Pronat), este ainda apresenta certos gargalos, como a definição de critérios para a escolha dos territórios. Neste sentido, o artigo apresenta metodologia estatística com o objetivo de avaliar os critérios de elegibilidade e o grau de importância desses critérios para que um município seja atendido pelo programa, a partir de indicadores proxies aos critérios definidos em decreto. Como resultado, critérios como densidade demográfica, existência de quilombos e população indígena não apresentaram o protagonismo esperado. Já o Índice de Desenvolvimento Humano apresentou-se como principal critério de elegibilidade, mostrando que o indicador se mantém relevante para o ciclo de monitoramento e avaliação de políticas públicas brasileiras. A agricultura familiar, um dos principais pilares do PTC e de seus programas antecessores, também se mostrou importante, mantendo a coerência do escopo e objetivos do programa.

Palavras-chaves: Territórios da cidadania; Política de desenvolvimento territorial; Critérios de elegibilidade; Modelo de regressão logístico.

Abstract: The Territories of Citizenship Program (PTC) is one of the most important territorial development policies in force in Brazil, especially regarding its scope and importance. The program features different scope, with territorial approach, social and inter-ministerial management. Despite the fact that the PTC is based in an evolutionary

1. Data de submissão: 14 de outubro de 2015. Data de aceite: 30 de junho de 2016.

2. Universidade Estadual de Campinas, Campinas, SP, Brasil. E-mail: pedrogandrade@yahoo.com.br

3. Escola Nacional de Ciências Estatísticas e Universidade do Estado do Rio de Janeiro, Rio de Janeiro, RJ, Brasil. E-mail: julia.strauch@ibge.gov.br

4. Escola Nacional de Ciências Estatísticas, Rio de Janeiro, RJ, Brasil E-mail: gustavo.ferreira@ibge.gov.br 
process of public policies, such as the National Program to Strengthen Family Agriculture (Pronaf) and Sustainable Development Program of Rural Areas (Pronat), it still presents certain bottlenecks, as the definition criteria for the choice of the territories. In this sense, the paper presents a statistical methodology with the objective of assessing the eligibility criteria and the importance level of these criteria for a municipality to be served by the program, from proxy indicators to the criteria set by decree. As a result, criteria such as population density, existence of quilombos and indigenous population did not show the expected role. But the Human Development Index is presented as the main eligibility criteria, showing that the index remains relevant for the monitoring and evaluation cycle of Brazilian public policy. Family farming, one of the main pillars of PTC and its predecessor programs, was also important, maintaining the consistency of the scope and objectives of the program.

Key-words: Territories of citizenship; Territorial development policy; Eligibility criteria; Logistic regression model.

DOI - http://dx.doi.org/10.1590/1234-56781806-94790540401

Classificação JEL: R58, O18.

\section{Introdução}

O debate atual sobre desenvolvimento rural no Brasil ganhou força a partir de movimentos sociais como o Movimento dos Trabalhadores Rurais Sem Terra (MST) no final dos anos 1980. O meio rural passou a ser percebido como uma esfera que vai muito além da agricultura, sendo necessário ao Estado compreender a dinâmica do meio rural e consequentemente, descobrir como interagem os diferentes atores sociais no território (ABRAMOVAY, 2010; SANTOS, 2011). Com esta nova perspectiva, existe uma mudança do debate não apenas para o desenvolvimento rural, mas para o desenvolvimento territorial (RÜCKERT, 2007; FAVARETO, 2010; DELGADO e LEITE, 2011).

Desde então, a gestão pública brasileira, vivenciada pós-constituinte de 1988, tem possibilitado maior participação da sociedade civil nos processos decisórios do Estado. Novos instrumentos de participação, relacionados ao controle e gestão das políticas sociais, foram estabelecidos, sobretudo através de canais que propiciaram o exercício da democracia participativa em políticas públicas de desenvolvimento territorial em áreas rurais (MELO et al., 2015). Esses canais participativos têm ajudado o Estado a compreender melhor as demandas locais e, mesmo ainda que por hipótese, tem possibilitado melhor direcionamento e alocações de suas ações. O conceito de desenvolvimento social, seja por temáticas mais específicas como combate à fome, combate à pobreza, ou valorização do trabalhador, tem sido assunto em pauta na gestão pública brasileira, ganhando força na agenda política desde meados da década de 1990.

Assim o desenvolvimento rural/territorial vem sendo tratado cotidianamente na pauta das agendas da gestão pública e está relacionado, entre outros aspectos, à pobreza rural e as desigualdades social e regional (FAVARETO, 2010; RÜCKERT, 2007; DELGADO e LEITE, 2011; ZIMMERMANN et al., 2014). Isto pode ser observado nos principais programas sociais voltados para políticas de desenvolvimento territorial no Brasil, a partir dos anos 1990. Ainda segundo Delgado e Leite (2011), a política de desenvolvimento territorial no meio rural representou um avanço considerável, mediante a experiência recente do País, apesar de diversos desafios que ainda se apresentam.

Destarte, a conjuntura atual das políticas públicas com enfoque territorial adotado pelo governo brasileiro, empregando o conceito de gestão social, tem por objetivo privilegiar a participação da sociedade e dos demais atores de um território, visando o alcance do desenvolvimento territorial sustentável. Exemplos deste tipo de abordagem são observados na evolução 
das políticas de desenvolvimento territorial adotadas pelo governo brasileiro, a saber: Programa Nacional de Fortalecimento da Agricultura Familiar (Pronaf); Programa de Desenvolvimento Sustentável dos Territórios Rurais (Pronat) e, contemporaneamente; no Programa Territórios da Cidadania (PTC) (OLIVEIRA et al., 2011); demonstrando, assim, a importância que vem sendo dada às políticas direcionadas às regiões rurais, com a existência de espaços participativos entre a sociedade e o Estado.

Cada vez mais este assunto tem se mostrado prioritário e os obstáculos existentes para a articulação de políticas, de instituições e a construção de estratégias de desenvolvimento devem ser superados. Desta forma, são necessários estudos para avaliação dessas políticas públicas, como um instrumento para o conhecimento da viabilidade de programas e projetos, e para o redirecionamento de seus objetivos, quando necessário, ou mesmo para a reformulação de suas propostas e atividades (CAVALCANTI, 2015). A avaliação é um mecanismo de gestão que fornece informações e subsídios para tomada de decisão dos gestores, formuladores e implementadores de programas, pois possibilita conhecer o que está acontecendo e atuar sobre os fatos de forma a realizar ajustes necessários.

Nesse sentido, foi escolhido o Programa Territórios da Cidadania, a política pública de desenvolvimento territorial mais importante na atualidade. O programa tem suas ações alocadas a territórios, denominados de "territórios da cidadania", que são aglomerados de municípios contíguos com características comuns (BRASIL, 2008). Esses territórios foram criados a partir dos territórios rurais, oriundos do Pronat, que se encontravam em maior situação de vulnerabilidade. De acordo com o decreto de 23 de março de 2009 (BRASIL, 2009), atualização do decreto de 25 de fevereiro de 2008 (decreto de implantação do PTC), alguns critérios de elegibilidade são definidos para um município pertencer a um dos 120 territórios. Dentre eles, os critérios mais tangíveis são: baixo IDH; baixa densidade demográ- fica; concentração de beneficiários do Programa Bolsa Família; concentração de pescadores; concentração de agricultura familiar e assentamentos da reforma agrária; concentração de populações quilombolas e indígenas e baixo Índice de Desenvolvimento da Educação Básica (IDEB).

$\mathrm{Na}$ literatura, a análise do Programa Territórios da Cidadania (PTC) apresenta duas abordagens. A primeira, uma perspectiva qualitativa que trata do aspecto teórico, abordando a participação e gestão social, conforme pode ser observado em estudos de Zani (2010), Melo (2011), Oliveira et al. (2011), Pereira et al. (2011), Lopes (2012), entre outros. A segunda, uma abordagem quantitativa de caráter empirista, que trata de territórios específicos, selecionados a priori, como pode ser observado em Tecchio e Cazella (2013), Lasa e Delgado (2013), Lopes et al. (2013), Barbosa et al. (2013), Rosar et al. (2013) e Zimmermann et al. (2014).

Todavia, existe a carência de estudos empíricos quantitativos que analisem o programa de forma global, abordando todos os 120 territórios do programa atual. Deste modo, este trabalho apresenta metodologia com o objetivo de analisar se os municípios atendem individualmente aos critérios de elegibilidade, a partir de uma abordagem empírica, através de análises descritivas e de um modelo estatístico (logístico). Além disso, a aplicação metodológica possibilita elencar o grau de importância de cada um dos critérios de elegibilidade no desenho dos territórios.

\section{Programa Territórios da Cidadania}

O PTC é um dos principais projetos correntes de desenvolvimento territorial do Brasil. Criado por decreto presidencial em 25 de fevereiro de 2008 e oriundo do Pronat, o programa tem abrangência nacional e seu objetivo é promover a redução das desigualdades sociais em áreas rurais, conforme ressalta o Art. $2^{\mathrm{o}}$ do decreto ilustrado no Quadro 1. 
Quadro 1. Decreto presidencial de 25/2/2008 - Programa Territórios da Cidadania

O PRESIDENTE DA REPÚBLICA, no uso da atribuição que lhe confere o art. 84, inciso VI, alínea "a", da Constituição, DECRETA:

Art. 1 o Fica instituído o Programa Territórios da Cidadania, a ser implementado de forma integrada pelos diversos órgãos do Governo Federal responsáveis pela execução de ações voltadas à melhoria das condições de vida, de acesso a bens e serviços públicos e a oportunidades de inclusão social e econômica às populações que vivem no interior do País.

$\S 1$ o Os Territórios da Cidadania serão criados e modificados pelo Comitê Gestor Nacional previsto no art. $6^{\circ}$, a partir dos agrupamentos municipais que apresentem densidade populacional média abaixo de oitenta habitantes por quilômetro quadrado e, concomitantemente, população média municipal de até cinquenta mil habitantes, com base nos dados censitários mais recentes. (Redação dada pelo Decreto de 24 de novembro de 2011)

$\S 2^{\circ}$ Os Municípios que compõem os Territórios da Cidadania serão agrupados segundo critérios sociais, culturais, geográficos e econômicos e reconhecidos pela sua população como o espaço historicamente construído ao qual pertencem, com identidades que ampliam as possibilidades de coesão social e territorial.

$\S 3^{\text {o }}$ São Territórios da Cidadania, sem prejuízo daqueles que forem instituídos na forma do $\S 1$ o , os agrupamentos de Municípios relacionados no Anexo a este Decreto.

Art. $2^{\circ}$ O Programa Territórios da Cidadania tem por objetivo promover e acelerar a superação da pobreza e das desigualdades sociais no meio rural, inclusive as de gênero, raça e etnia, por meio de estratégia de desenvolvimento territorial sustentável que contempla:

I - integração de políticas públicas com base no planejamento territorial;

II - ampliação dos mecanismos de participação social na gestão das políticas públicas de interesse do desenvolvimento dos territórios;

III - ampliação da oferta dos programas básicos de cidadania;

IV - inclusão e integração produtiva das populações pobres e dos segmentos sociais mais vulneráveis, tais como trabalhadoras rurais, quilombolas, indígenas e populações tradicionais;

V - valorização da diversidade social, cultural, econômica, política, institucional e ambiental das regiões e das populações.

Art. 3o A escolha e priorização do território a ser incorporado ao Programa Territórios da Cidadania dar-se-ão pela ponderação dos seguintes critérios:

I - estar incorporado ao Programa Desenvolvimento Sustentável de Territórios Rurais, do Ministério do Desenvolvimento Agrário;

II - menor Índice de Desenvolvimento Humano - IDH territorial;

III - maior concentração de beneficiários do Programa Bolsa Família;

IV - maior concentração de agricultores familiares e assentados da reforma agrária;

V - maior concentração de populações tradicionais, quilombolas e indígenas;

VI - baixo dinamismo econômico, segundo a tipologia das desigualdades regionais constantes da Política Nacional de Desenvolvimento Regional, do Ministério da Integração Nacional;

VII - convergência de programas de apoio ao desenvolvimento de distintos níveis de governo; (Redação dada pelo Decreto de 23 de março de 2009)

VIII - maior organização social; e (Redação dada pelo Decreto de 23 de março de 2009)

IX - maior concentração de municípios de menor IDEB - Índice de Desenvolvimento de Educação Básica. (Incluído pelo Decreto de 23 de março de 2009) Parágrafo único. O critério descrito no inciso IX será utilizado para a incorporação de Territórios a partir de 2009. (Incluído pelo Decreto de 23 de março de 2009).

Fonte: Brasil (2008).

O programa prioriza a inclusão produtiva das populações pobres dos territórios, a busca da universalização de programas básicos de cidadania, o planejamento e integração de políticas públicas e a ampliação da participação social. Deste modo, o PTC tem ações vinculadas a três eixos de atuação:
1. Apoio a Atividades Produtivas;
2. Cidadania e Direito;
3. Infraestrutura.

Estes eixos se desdobram em sete temas:

1. Organização Sustentável da Produção;

2. Ações Fundiárias;

3. Educação e Cultura;

4. Direitos e Desenvolvimento Social;

5. Saúde, Saneamento e Acesso à Água;

6. Apoio à Gestão Territorial;

7. Infraestrutura 5 .

5. Disponível em: < http://www.territoriosdacidadania.gov.br/>. Acesso em: 10 jul. 2014. 
Figura 1. Municípios pertencentes a Territórios da Cidadania

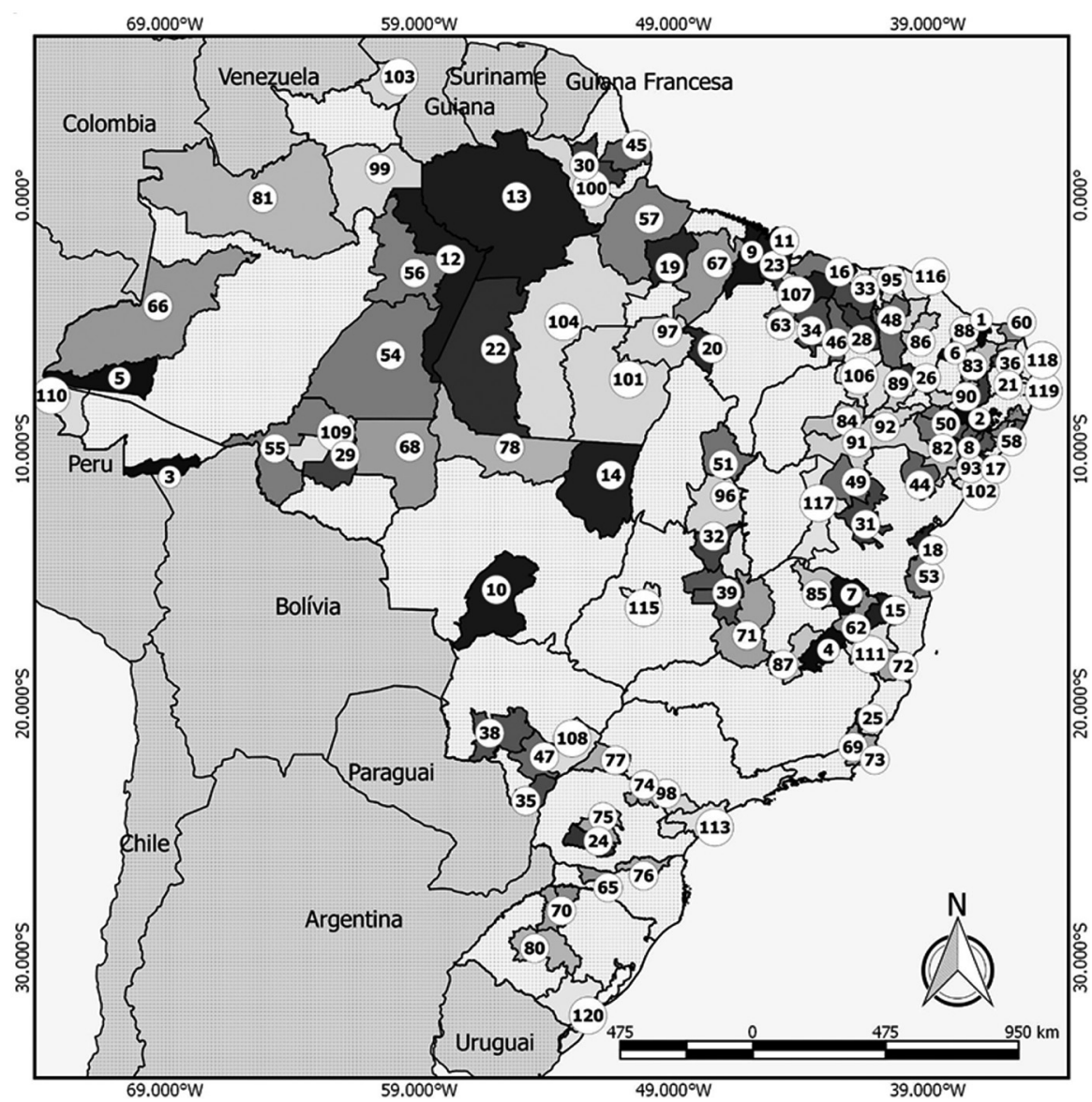

\begin{tabular}{|c|c|c|c|}
\hline ID - Nome do território & ID - Nome do território & ID - Nome do território & ID - Nome do território \\
\hline 1-Açu-Mossoró - RN & 31-Chapada Diamantina - BA & 61-Médio Alto Uruguai - RS & 91-Sertão do São Francisco - BA \\
\hline 2-Agreste Meridional - PE & 32-Chapada dos Veadeiros - GO & 62-Médio Jequitinhonha - MG & 92-Sertão Do São Francisco - PE \\
\hline 3-Alto Acre e Capixaba - AC & 33-Cocais - MA & 63-Médio Mearim - MA & 93-Sertão Ocidental - SE \\
\hline 4-Alto Jequitinhonha-MG & 34-Cocais - PI & 64-Médio Sertão - PB & 94-Sertões De Canindé - CE \\
\hline 5-Alto Juruá - AM & 35-Cone Sul-MS & 65-Meio Oeste Contestado - SC & 95-Sobral-CE \\
\hline 6-Alto Oeste - RN & 36-Curinataú-PB & 66-Mesorregião Alto Solimôes - AM & 96-Sudeste-TO \\
\hline 7-Alto Rio Pardo-MG & 37-Da Bacia Leiteira - AL & 67-Nordeste Paraense - PA & 97-Sudeste Paraense - PA \\
\hline 8-Alto Sertão-SE & 38-Da Reforma - MS & 68-Noroeste-MT & 98-Sudoeste Paulista-SP \\
\hline 9-Alto Turi e Gurupi - MA & 39-Das Águas Emendadas - DF,GO,MG & 69-Noroeste - RJ & 99-Sul De Roraima - RR \\
\hline 10-Baixada Cuiabana - MT & 40-Do Agreste - AL & 70-Noroeste Colonial - RS & 100-Sul Do Amapá - AP \\
\hline 11-Baixada Ocidental-MA & 41-Do Alto Sertão - AL & 71-Noroeste De Minas - MG & 101-Sul Do Pará,Alto Xingu - PA \\
\hline 12-Baixo Amazonas - AM & 42-Do Litoral Norte - AL & 72-Norte - ES & 102-Sul Sergipano - SE \\
\hline 13-Baixo Amazonas - PA & 43-Do Médio Sertão - AL & 73-Norte - RJ & 103-Terra Indígena Raposa Serra do Sol e São Marcos - RR \\
\hline 14-Baixo Araguaia - MT & 44-Do Sisal - BA & 74-Norte Pioneiro - PR & 104-Transamazônica - PA \\
\hline 15-Baixo Jequitinhonha - MG & 45-Dos Lagos - AP & 75-Paraná Centro - PR & 105-Vale do Canindé - PI \\
\hline 16-Baixo Parnaíba-MA & 46-Entre Rios - PI & 76-Planalto Norte-SC & 106-Vale Do Guaribas - PI \\
\hline 17-Baixo São Francisco - SE & 47-Grande Dourados - MS & 77-Pontal Do Paranapanema - SP & 107-Vale Do Itapecuru - MA \\
\hline 18-Baixo Sul - BA & 48-Inhamuns Crateús - CE & 78-Portal Da Amazônia - MT & 108-Vale do Ivinhema - MS \\
\hline 19-Baixo Tocantins - PA & 49-Irecê- BA & 79-Potengi-RN & 109-Vale Do Jamarí - RO \\
\hline 20-Bico Do Papagaio - TO & 50-Itaparica - BA,PE & 80-Região Central - RS & 110-Vale Do Juruá - AC \\
\hline 21-Borborema-PB & 51-Jalapão-TO & 81-Rio Negro da Cidadania Indígena - AM & 111-Vale Do Mucuri-MG \\
\hline 22-BR $163-\mathrm{PA}$ & 52-Lençóis Maranhenses,Munin - MA & 82-Semi-árido Nordeste II - BA & 112-Vale do Paranã - GO \\
\hline 23-Campo e Lagos - MA & 53-Litoral Sul - BA (Antigo Sul) & 83-Seridó - RN & 113-Vale Do Ribeira - PR \\
\hline 24-Cantuquiriguaçu - PR & 54-Madeira - AM & 84-Serra Da Capivara - PI & 114-Vale Do Ribeira - SP \\
\hline 25-Caparaó - ES & 55-Madeira Mamoré - RO & 85-Serra Geral -MG & 115-Vale Do Rio Vermelho - GO \\
\hline 26-Carirí-CE & 56-Manaus e Entorno - AM & 86-Sertão Central - CE & 116-Vales do Curu e Aracatiaçu - CE \\
\hline 27-Cariri Ocidental - PB & 57-Marajó - PA & 87-Sertão de Minas - MG & 117-Velho Chico - BA \\
\hline 28-Carnaubais - PI & 58-Mata Alagoana - AL & 88-Sertão Do Apodi - RN & 118-Zona Da Mata Norte - PB \\
\hline 29-Central - RO & 59-Mata Sul-PE & 89-Sertão do Araripe - PE & 119-Zona Da Mata Sul - PB \\
\hline 30-Centro Oeste - AP & 60-Mato Grande - RN & 90-Sertão Do Pajeú - PE & 120-Zona Sul Do Estado - RS \\
\hline
\end{tabular}

Fonte: Ministérios do Desenvolvimento Agrário (MDA), Instituto Brasileiro de Geografia e Estatística (IBGE), 2008/2009. 
O Programa tem como proposta uma organização democrática participativa, através de colegiados e cooperação solidária.

O PTC iniciou-se tomando como critério os territórios rurais economicamente mais fragilizados, sendo territórios com menores índices de desenvolvimento humano (IDH), com a maior concentração de agricultores familiares ou assentados da reforma agrária, com maior concentração de quilombolas ou população indígenas e também com territórios de uma boa parte de municípios com baixo dinamismo econômico (BRASIL, 2008). Até 2015 o programa contempla 1.852 municípios, distribuídos entre os 120 territórios (Figura 1). A criação destes territórios foi feita em dois momentos distintos, 60 territórios criados no ano de implantação do programa (2008) e 60 no ano seguinte (2009).

Destes municípios, Boa Vista, em Roraima, não é contemplado inteiramente pelo programa, mas apenas uma pequena parte do seu território.

\section{Materiais e métodos}

Para o desenvolvimento deste trabalho, inicialmente, foram analisados os artigos do decreto de implantação do PTC de modo a compreender os critérios de elegibilidade do Programa dos Territórios da Cidadania. Posteriormente, foram selecionadas as variáveis e o método de modelagem mais adequado.

\subsection{Materiais}

A seleção das variáveis foi realizada a partir de um estudo sobre fontes de dados disponíveis e de variáveis que pudessem ser consideradas como proxies para identificar fatores associados aos critérios de elegibilidade definidos nos artigos $1^{\text {o }}$ e $3^{\text {o }}$ do decreto, conforme apresentado no Quadro 2. Cabe ressaltar que a maior parte das variáveis selecionadas mensura diretamente o

Quadro 2. Variáveis selecionadas de acordo com os critérios de elegibilidade

\begin{tabular}{|c|c|c|c|c|}
\hline Variável & Descrição & Fonte & Ano & \begin{tabular}{|c|} 
№ de \\
municípios
\end{tabular} \\
\hline TERRITÓRIO & $\begin{array}{l}\text { Variável binária que indica os municípios que } \\
\text { pertencem a um dos } 120 \text { territórios da cidadania }\end{array}$ & Decreto & 2009 & 5.565 \\
\hline IDHM & Índice de Desenvolvimento Humano Municipal & $\begin{array}{l}\text { Programa das Nações Unidas para } \\
\text { o Desenvolvimento }\end{array}$ & 2010 & 5.565 \\
\hline F_PBF & $\begin{array}{l}\text { Número de famílias beneficiárias do programa } \\
\text { Bolsa Família }\end{array}$ & \begin{tabular}{|l|}
$\begin{array}{l}\text { Ministério do Desenvolvimento } \\
\text { Social e Combate à Fome }\end{array}$ \\
\end{tabular} & 2010 & 5.565 \\
\hline TX_RURAL & Percentual da população rural & $\begin{array}{l}\text { Instituto Brasileiro de Geografia e } \\
\text { Estatística }\end{array}$ & 2010 & 5.565 \\
\hline TXMIL_INDIGENA & Número de índios a cada mil habitantes & $\begin{array}{l}\text { Instituto Brasileiro de Geografia e } \\
\text { Estatística }\end{array}$ & 2010 & 5.565 \\
\hline DENS_DEM & Densidade demográfica & $\begin{array}{l}\begin{array}{l}\text { Instituto Brasileiro de Geografia e } \\
\text { Estatística }\end{array} \\
\end{array}$ & 2010 & 5.565 \\
\hline PO_AGRICULT_F & $\begin{array}{l}\text { Pessoas ocupadas em agricultura familiar (pessoas } \\
\text { de } 14 \text { anos ou mais) }\end{array}$ & $\begin{array}{l}\text { Instituto Brasileiro de Geografia e } \\
\text { Estatística }\end{array}$ & 2006 & 5.548 \\
\hline QUILOMBO & $\begin{array}{l}\text { Variável binária que indica os municípios que } \\
\text { possuem pelo menos uma comunidade (reco- } \\
\text { nhecida) remanescente de Quilombos }\end{array}$ & Fundação Cultural Palmares & 2010 & 5.565 \\
\hline IDEB_4* & $\begin{array}{l}\text { Índice de Desenvolvimento da Educação Básica } \\
\text { da rede pública, séries iniciais ( } 4^{\mathrm{a}} \text { série) }\end{array}$ & \begin{tabular}{|l|}
$\begin{array}{l}\text { Instituto Nacional de Estudos e Pes- } \\
\text { quisas Educacionais Anísio Teixeira }\end{array}$ \\
\end{tabular} & 2009 & $5.513^{*}$ \\
\hline IDEB_8* & $\begin{array}{l}\text { Índice de Desenvolvimento da Educação Básica } \\
\text { da rede pública, séries finais ( } 8^{\underline{a}} \text { série) }\end{array}$ & \begin{tabular}{|l|}
$\begin{array}{l}\text { Instituto Nacional de Estudos e Pes- } \\
\text { quisas Educacionais Anísio Teixeira }\end{array}$ \\
\end{tabular} & 2009 & $5.546^{4}$ \\
\hline NUM_FAM_ASS & $\begin{array}{l}\text { Número de famílias assentadas. Significa núme- } \\
\text { ro de lotes ocupados. Em um mesmo lote podem } \\
\text { morar várias famílias }\end{array}$ & $\begin{array}{l}\text { Ministério do Desenvolvimento } \\
\text { Agrário }\end{array}$ & $\begin{array}{l}2003 / \\
2009\end{array}$ & 5.565 \\
\hline PESCADORES & $\begin{array}{l}\text { Número de pescadores (pessoas de } 10 \text { anos ou } \\
\text { mais) }\end{array}$ & $\begin{array}{l}\text { Instituto Brasileiro de Geografia e } \\
\text { Estatística }\end{array}$ & 2010 & 5.565 \\
\hline
\end{tabular}

* Considerando as projeções feitas pelo Inep.

Fonte: Elaboração própria. 
que foi proposto no decreto de implantação do Programa Territórios da Cidadania ou são citadas em apresentações e relatórios do $\mathrm{MDA}^{6}$.

Para a data de referência do estudo foi levada em consideração a disponibilidade dos dados em anos próximos da criação dos territórios da cidadania, ou seja, 2008 e 2009. Cabe ressaltar que, para o número de famílias assentadas, foi construída uma variável de estoque, com período de contagem que engloba o ano de implantação do Pronat e o último ano no qual foram criados os territórios da cidadania, a saber, 2003 e 2009.

Para a elaboração do trabalho foi utilizado o software R v3.0.2 com seus pacotes básicos e alguns pacotes específicos de sua biblioteca, a saber: para manipulação da base de dados $\left(\right.$ pacote foreign $\left.{ }^{7}\right)$, tabulação, estimação de modelos estatísticos (pacotes: $\mathrm{rms}^{8}$ e gmodels ${ }^{9}$ ) e imputação via regressão. O software TerraView v4.2.2 foi utilizado para imputação via estimador bayesiano empírico. Por fim, foi utilizado o software QGis v2.0.1 para a criação dos mapas temáticos e criação/adequação de malhas territoriais - todos são softwares livres.

\subsection{Métodos para imputação de dados}

Após a construção da base de dados, foi verificado que, apesar de não ter sido criado nenhum município nos anos considerados para as variáveis, algumas não apresentavam dados para todos os 5.565 municípios brasileiros. Analisando os municípios que possuíam dados para todas as variáveis, a perda de municípios seria de menos de $1 \%$, resultando em base de dados com total de 5.513 municípios. Esta base de dados foi reservada e outra foi criada, realizando-se a imputação das variáveis que possuem menor número de informações, a saber: os IDEBs e o número de pessoas envolvidas em agricultura familiar. A opção de realizar a imputação foi basicamente devido

6. Disponível em: < http://www.territoriosdacidadania.gov.br/>. Acesso em: 10 jul. 2014.

7. R Core Team (2013).

8. Harrell (2013).

9. Warnes et al. (2013). ao interesse em obter estimativas para todos os municípios brasileiros.

A variável "PO_AGRICULT_F", pessoas ocupadas em agricultura familiar, foi imputada através da informação de seus vizinhos de primeira ordem, ou seja, os municípios que eram fronteiriços à região de interesse, utilizando-se a técnica do estimador bayesiano empírico. Esta técnica, em geral, é utilizada para estimar, suavizar ou corrigir medidas em que o numerador seja a população que sofreu certo evento e, o denominador, a população exposta ao risco, conforme observado em Marshall (1991).

Para os IDEB da $4^{\mathrm{a}}$ e $8^{\mathrm{a}}$ séries, foi utilizada outra técnica de imputação, através de um modelo de regressão simples. A mudança da técnica deve-se à própria natureza da variável e por se acreditar que ela não possua correlação com o espaço geográfico, mas, sim, com outros indicadores, que é o caso do Índice de Desenvolvimento Humano, dimensão educação. Ambas as regressões obtiveram o teste $F$ significativo e com proporção da variabilidade explicada de $44,0 \%$ para IDEB da $4^{\text {a }}$ série e $37,4 \%$ para o IDEB da 8aㅗ série.

Ressalta-se que, no desenvolvimento do trabalho, optou-se por não considerar Boa Vista como um município pertencente a um território da cidadania, pois apenas um de seus setores censitários faz parte do território; deste modo, o estudo será feito considerando 1.851 municípios, ao invés dos 1.852 totalizados em decreto.

\subsection{Modelo logístico}

O modelo escolhido para realizar este trabalho foi o modelo logístico, usando a variável dicotômica TERRITORIO. Trata-se de um modelo pertencente à família exponencial, do grupo de modelos lineares generalizados (MLG).

Os MLGs são caracterizados por três componentes: a componente aleatória, um conjunto de variáveis explicativas e uma função de ligação. A componente aleatória é formada pelo vetor de observações $Y$, que deve ser uma variável aleatória com distribuição de probabilidade pertencente à família exponencial (DOBSON e 
BARNETT, 2002). Neste estudo, tem-se $Y$, uma variável dicotômica, que possui dois valores possíveis, "1" para os municípios pertencentes a um dos 120 territórios da cidadania e " 0 " em caso contrário. Neste caso, $Y_{i} \sim B E R\left(\pi_{i}\right)$ e $\operatorname{Pr}\left(Y_{i}=1\right)=\pi$ e $\operatorname{Pr}\left(Y_{i}=0\right)=1-\pi$.

Segundo Dobson e Barnett (2002), a componente sistemática é composta pelas variáveis explicativas, neste caso, associada aos critérios de elegibilidade, que representa a estrutura linear do modelo, em que as $p$ covariáveis $\left(x_{1}, \ldots, x_{p}\right)$ formam um preditor linear $\eta$ tal que $\eta_{i}=x_{i}^{T} \beta$ e $\beta$ representa o vetor de parâmetros a serem estimados. A função de ligação entre a componente aleatória e sistemática no caso do modelo estudado é a logit, a saber:

$$
g\left(\neq_{i}\right)=\eta_{i}=\log i t \neq_{i}=\ln \left(\neq_{i} / 1-\neq_{i}\right)
$$

De acordo com Demaris (1992), o modelo logístico segue alguns pressupostos:

a) a regressão logística não assume uma relação linear entre a variável dependente e variáveis independentes;

b) a variável dependente deve ser dicotômica;

c) as variáveis independentes não precisam ser intervalares, nem linearmente relacionadas, nem com igualdade de variância dentro de cada grupo;

d) as categorias (grupos) devem ser mutuamente exclusivas.

Para verificar a associação entre as variáveis regressoras e a chance de determinado evento acontecer, como por exemplo, a probabilidade de um município pertencer ao Programa Territórios da Cidadania, deve-se primeiro avaliar a inclusão de cada variável regressora no modelo estatístico. Em outras palavras, avalia-se se a inserção no modelo de variáveis associadas aos critérios de elegibilidade ajuda a aumentar o seu poder explicativo.

Após realizar o ajuste do modelo, deve-se testar individualmente a significância estatística de cada um dos parâmetros do modelo. Neste caso, testam-se as seguintes hipóteses: $\mathrm{H}_{0}: \beta_{j}=0$ versus $\mathrm{H}_{1}: \beta_{j} \neq 0$, utilizando-se a estatística de Wald $(W)$, a saber:

$$
W=\frac{\left(\hat{\beta}_{j}\right)^{2}}{\sqrt{\sigma^{2}\left(\hat{\beta}_{j}\right)}}
$$

e rejeita-se $H_{0}$ se somente se $W>\chi_{(1)}^{2}$, dado um nível de significância $\alpha$ (DOBSON e BARNETT, 2002).

Uma forma alternativa de avaliar modelos é a medida estatística chamada de AIC. O critério de informação de Akaike (AIC) é uma medida da qualidade que possibilita a comparação simultânea de dois ou mais modelos. O AIC não possui um intervalo limitado e menores valores de AIC indicam melhor qualidade do ajuste do modelo. Outra medida para avaliar a qualidade do ajuste é o pseudo $R^{2}$. Existem várias formulações para esta medida, sendo a mais comum o Nagelkerke $R^{2}$ proposto em 1991 (UNIVERSITY OF CALIFORNIA, 2011).

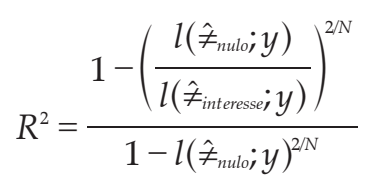

Esta medida, assim como suas variações, tem o objetivo de mensurar a proporção da variabilidade explicada pelo modelo, que pode variar no intervalo $[0,1]$.

Cabe ressaltar que, neste artigo, o interesse era apenas a avaliação individual dos critérios de elegibilidade; desta forma, foram ajustados apenas modelos de regressão com apenas uma única variável explicativa. Assim, o intuito é avaliar os critérios de elegibilidade, verificando a existência de associação entre cada um dos critérios e a chance de um município pertencer ao PTC.

\section{Resultados e discussão}

A análise exploratória dos dados mostrou que o Programa Territórios da Cidadania, de acordo com dados do Censo 2010, cobria 45,8\% da população rural brasileira, correspondendo a 
13.7 milhões de pessoas. O programa, ainda de acordo com dados do Censo 2010, atuava em territórios em que residiam cerca de 410 mil índios, correspondendo a 49,86\% da população indígena brasileira.

De acordo com dados do Ministério de Desenvolvimento Social e Combate à Fome, em 2010, cerca de 4,66 milhões de famílias residentes dos territórios da cidadania recebiam Bolsa Família, correspondendo a apenas 37,5\% do total de famílias beneficiárias do País.

O Índice de Desenvolvimento Humano médio dos municípios atendidos pelo programa era de 0,62 em 2010, enquanto que, nos demais municípios, era de 0,68 . Considerando os municípios que registraram baixo desenvolvimento humano (inferior a 0,49), conforme tipologia do PNUD, 31 municípios estavam nesta situação, mas apenas 18 deles eram atendidos pelo PTC. De acordo com dados do Instituto de Pesquisas Educacionais Anísio Teixeira, o valor médio do Índice de Desenvolvimento da Educação Básica (Ideb) dos municípios pertencentes aos territórios da cidadania, para alunos da rede pública da quarta série, era 4 e, no restante do País, 4,8. Já para alunos da $8^{\text {a }}$ série, o Ideb era de 3,5 nos municípios pertencentes a territórios da cidadania e 3,9 para os não pertencentes.

Em relação ao número de pescadores, de acordo com dados censitários de 2010, cerca de 230 mil pescadores residiam em municípios pertencentes aos territórios da cidadania, correspondendo a $56,2 \%$ da população de pescadores do País. De acordo com o Censo Agropecuário de 2006, cerca de 6,4 milhões de pessoas de 14 anos ou mais estavam ocupadas em atividades de agricultura, representando $49,1 \%$ dos trabalhadores familiares do Brasil. De acordo com dados do Ministério Agrário, no período entre 2003 e 2009, 239.698 famílias foram assentadas em territórios da cidadania, correspondendo a 68,3\% do total nacional. Observando os dados da Fundação Cultural Palmares, observa-se que 393 municípios que pertenciam a territórios da cidadania possuíam pelo menos uma comunidade quilombola certificada, representando $54,5 \%$ do total de municípios que possuíam pelo menos uma comunidade.

Deste modo, nesta análise preliminar, verificou-se que há evidências empíricas de tendências de conformidade com o decreto de implantação do programa. A seguir, foram realizadas análises individuais dos critérios de elegibilidade dos Artigos 1 e 3.

\subsection{Artigo $1^{\circ}$, parágrafo 1}

O $\S 1$ do artigo $1^{o}$ do decreto de implantação do PTC prevê que os territórios da cidadania seriam criados pelo Comitê Gestor Nacional, a partir dos agrupamentos municipais que apresentassem densidade populacional média abaixo de 80 habitantes por quilômetro quadrado e, concomitantemente, população média municipal de até 50 mil habitantes, com base nos dados censitários mais recentes (Quadro 1). Em 2009 existiu uma alteração no decreto que estabelece que passa a ser responsabilidade do Comitê Gestor não só a criação dos territórios, mas também sua modificação.

A análise da densidade populacional média dos territórios da cidadania empregou os dados do Censo Demográfico 2000, censo mais recente até o ano de implantação do programa (2008). De acordo com a Figura 2, verifica-se que existiam sete territórios dentre os 120 que não atendiam a esse critério de elegibilidade da densidade populacional média, a saber: Baixo São Francisco (SE), criado em 2009; Borborema (PB), criado em 2008; Carirí (CE), criado em 2009; Do Agreste (AL), criado em 2008; Mata Sul (PE), criado em 2008; Zona da Mata do Norte (PB), criado em 2008; e Zona da Mata Sul (PB), criado em 2008. Deste modo, mesmo se fossem considerados apenas os 60 territórios iniciais, este critério de elegibilidade já não estava em conformidade em cinco dos 60 territórios.

Analisando a população média municipal de até 50 mil habitantes, também utilizando os dados do censo de 2000, pode-se observar na Figura 3 que existiam oito territórios dentre os 120 que não atendiam a esse critério, a saber: Baixada 
Figura 2. Avaliação do critério de densidade populacional média inferior a $80 \mathrm{hab} / \mathrm{km}^{2}$, Brasil, 2000
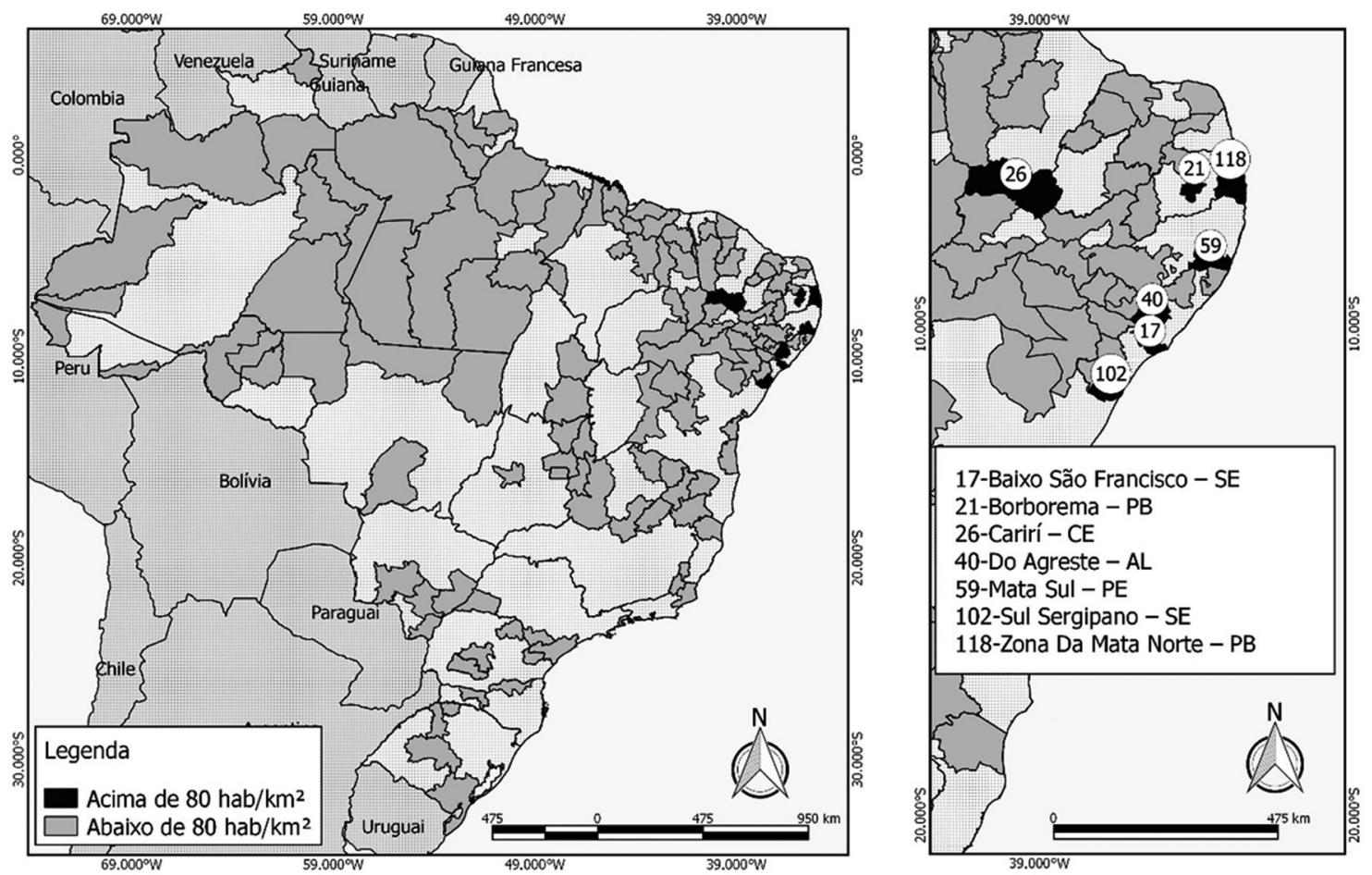

Fonte: Elaborado pelos autores com base nos dados do Censo Demográfico 2000, IBGE.

Figura 3. Avaliação do critério de população média municipal de até 50.000 hab, Brasil, 2000

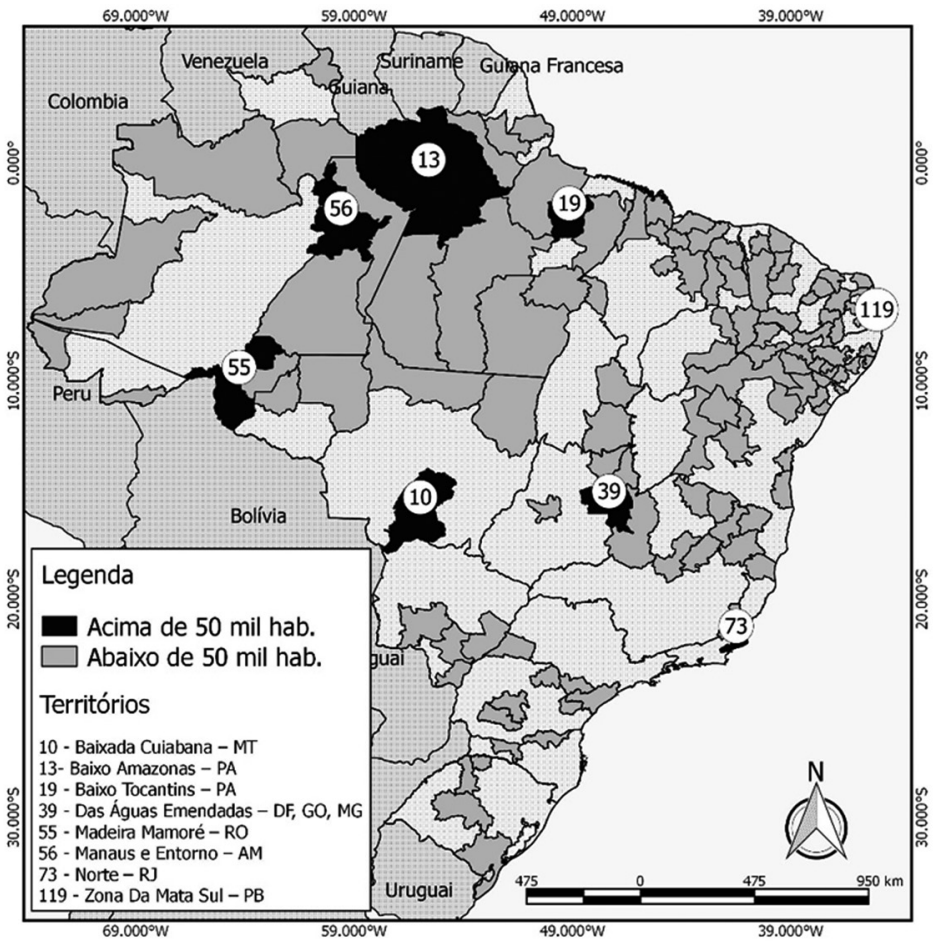

Fonte: Elaborado pelos autores com base nos dados do Censo Demográfico 2000, IBGE. 
Cuiabana (MT), criado em 2009; Baixo Amazonas (PA), criado em 2008; Baixo Tocantins (PA), criado em 2009; Das Águas Emendadas (DF, GO, MG), criado em 2008; Madeira Mamoré (RO), criado em 2009; Manaus e Entorno (AM), criado em 2008; Norte (RJ), criado em 2008 e Zona da Mata Sul (PB), criado em 2008. Deste modo, mesmo se fossem considerados apenas os 60 territórios iniciais, este critério de elegibilidade já não estaria em conformidade em cinco dos 60 territórios. Cabe ressaltar que o território Zona da Mata Sul (PB), criado no ano da implantação do programa, não possuía nem população média abaixo de 80 hab./ $\mathrm{km}^{2}$ e nem população média municipal de até 50 mil habitantes.

Nesse sentido, após a verificação empírica, foi constatado que nem todos os territórios da cidadania criados eram elegíveis de acordo com os critérios apresentados no $\S 1$ do artigo $1^{\mathrm{O}}$. Os $\S^{\mathrm{o}} \mathrm{e}$ $\S 3^{\mathrm{o}}$ não serão avaliados neste trabalho, pois o $\$ 2^{\mathrm{o}}$ trata de questões mais subjetivas e o $§ 3^{-}$não trata de critérios de elegibilidade, conforme pode ser verificado no Quadro 1.

\subsection{O artigo $3^{\circ}$ e seus incisos}

$\mathrm{O}$ artigo $3^{\circ}$, que se mantém inalterado desde a implantação do PTC, apresenta os critérios de elegibilidade para um município pertencer ao programa. O Inciso I pode ser avaliado realizando a correspondência entre os territórios da cidadania e os territórios rurais. O site do Sistema de Gestão Integrada (http://sge.mda.gov.br/), vinculado ao portal do Ministério do Desenvolvimento Agrário (MDA), apresenta informações a respeito dos territórios rurais e, consequentemente, da cidadania. Neste site, é possível obter "shapefiles" e planilhas em formato "xls" dos 164 territórios rurais e dos 120 territórios da cidadania, implantados até 2009 .

Após a verificação da compatibilidade entre as duas informações, observou-se que o território da cidadania intitulado Mesorregião Alto Solimões, situado no extremo oeste do estado do Amazonas, não pertencia à listagem de territórios rurais. Após extensa investigação, acredita-se que este território tenha sido criado no período entre 2006 e 2012, pois, de acordo com o MDA, até 2012 havia 165 territórios rurais; em 2013 foram criados mais 74 territórios rurais e neles não constavam o território Mesorregião Alto Solimões. Outro ponto de inconsistência é que a malha territorial dos territórios rurais foi construída com 164 territórios e em nenhum momento da história do Pronat foram totalizados 164, mas, sim, 165 territórios rurais. Deste modo, talvez tenha havido alguma defasagem na construção das informações apresentadas no "shape" e na planilha de territórios rurais. Como conclusão pode-se constatar que todos os 120 territórios da cidadania eram territórios rurais.

Os Incisos II, III, IV, V e IX podem ser avaliados de forma empírica através de regressões logísticas simples, utilizando cada critério de elegibilidade separadamente e verificando a significância dos parâmetros estimados em cada regressão. A análise individual dos critérios de elegibilidade é relevante, pois nem sempre um município atenderá concomitantemente a todos os critérios e esta é uma forma de verificar se de fato existe associação na escolha de territórios e os critérios. Além disso, é possível investigar o grau de relevância de cada um dos critérios de elegibilidade.

A partir da análise da distribuição dos indicadores, pode-se observar que as variáveis Famílias Beneficiárias do Programa Bolsa Família (F_ PBF), População Indígena a cada mil habitantes (TXMIL_INDIGENA), Densidade Demográfica (DENS_DEM), Pessoas Ocupadas envolvidas em Agricultura Familiar (PO_AGRICULT_F), Número de Famílias Assentadas (NUM_FAM ASS) e Número de Pescadores (PESCADORES) são assimétricas e possuem valores atípicos. Analisando os dados da Tabela 1, pode-se observar que elas possuem média distante da mediana, alta assimetria e presença de valores extremos e isto poderia influenciar bastante a modelagem. Desta forma, verificou-se a possibilidade de se trabalhar com variáveis transformadas. 
Tabela 1. Medidas resumo dos indicadores correspondentes aos critérios de elegibilidade

\begin{tabular}{lccccccc}
\hline \multicolumn{1}{c}{ Indicador } & Média & Mediana & Mínimo & Máximo & Assimetria & Desvio Padrão & Coef. de Variação \\
\hline DENS_DEM & 108,20 & 24,37 & 0,13 & 13024,56 & 13,6 & 572,44 & 5,29 \\
F_PBF & 2296,18 & 1006 & 1 & 196699 & 17,0 & 6926,08 & 3,02 \\
IDHM & 0,66 & 0,66 & 0,42 & 0,86 & $-0,2$ & 0,07 & 0,11 \\
PESCADORES & 74,31 & 0 & 0 & 7205 & 9,4 & 291,14 & 3,92 \\
PO_AGRICULT_F & 2344,76 & 1364 & 0 & 39015 & 3,2 & 2884,83 & 1,23 \\
NUM_FAM_ASS & 63,08 & 0 & 0 & 14233 & 17,4 & 364,52 & 5,78 \\
IDEB_4 & 4,51 & 4,60 & 0,5 & 8,6 & 0,1 & 1,00 & 0,22 \\
IDEB_8 & 3,79 & 3,80 & 1,6 & 6,6 & 0,0 & 0,73 & 0,19 \\
TX_RURAL & 36,17 & 35,34 & 0 & 95,82 & 0,2 & 22,04 & 0,61 \\
TXMIL_INDIGENA & 7,23 & 0,41 & 0 & 885,61 & 10,9 & 43,64 & 6,03 \\
\hline
\end{tabular}

Fonte: Elaborado pelos autores com base nos dados do Atlas do Desenvolvimento Humano (PNUD, 2013), MDS (2010), Censo Demográfico 2010 (IBGE) Censo Agropecuário 2006 (IBGE), INEP (2009), MDA (2010) e Fundação Cultural Palmares (2010).

Antes de verificar a relevância de se aplicar transformações nas variáveis era necessário avaliar o impacto da imputação na análise de resultados. Foi constatado que os resultados sem imputação são muitos próximos dos com imputação, indicando que a estimação dos valores faltantes não modificaria os resultados. Em relação às transformações, constatou-se que seria necessário suavizar a distribuição de alguns indicadores, pois o ajuste de algumas regressões resultou em baixos valores de pseudo $R^{2}$ e não significância de parâmetros. Desta forma, optou-se por trabalhar com as variáveis transformadas.

Nos casos em que o valor mínimo das variáveis era zero foi somado 1 unidade antes de aplicar o logaritmo natural, devido à descontinuidade desta função em zero. Observou-se que houve aumento de pseudo $R^{2}$ em todos os modelos e diminuição de AIC nos modelos em que foram aplicadas as transformações. Os modelos com menores AIC foram escolhidos como mais adequados para avaliar a associação com os critérios de elegibilidade, identificando, assim, a transformação mais adequada.

A Tabela 2 apresenta os coeficientes e estatísticas obtidos após o ajuste dos modelos finais, os quais englobam as imputações e as transformações nas variáveis. De acordo com a tabela, os testes de Wald indicaram que todas as variáveis foram significativas ao nível de 5\% de significância. Nesse sentido, há evidências de que estas variáveis possuam associação com os critérios de elegibilidade definidos em decreto, quando estes critérios são avaliados individualmente. Analisando-se os coeficientes de regressão das variáveis, em cada um dos modelos, pode-se observar que:

- Quanto menor a densidade demográfica dos municípios, maior é chance de pertencer a um território da cidadania;

- O mesmo ocorre para o IDH e Ideb, variáveis que possuem coeficiente negativo e significativo a um nível de 5\%;

- O número de famílias beneficiárias no programa Bolsa Família, número de pescadores, número de pessoas envolvidas em agricultura familiar, número de famílias assentadas, o percentual da população residente em áreas rurais e o número de índios a cada 1.000 habitantes têm coeficiente positivo, indicando que existe uma relação direta entre estas variáveis e a chance de um município pertencer a algum território da cidadania;

- A existência de quilombos também é um fator que aumenta a chance de um município pertencer a algum território da cidadania. 
Tabela 2. Coeficientes dos modelos de regressão logística simples escolhidos para a probabilidade de um município pertencer a um território da cidadania

\begin{tabular}{|c|c|c|c|c|c|c|c|}
\hline \multirow{2}{*}{ Variáveis } & \multirow{2}{*}{$\hat{\beta}$} & \multirow{2}{*}{$\begin{array}{c}\text { Erro } \\
\text { padrão }\end{array}$} & \multicolumn{3}{|c|}{ Teste de Hipótese } & \multirow{2}{*}{$\begin{array}{c}\text { Pseudo } \\
\mathbf{R}^{2}\end{array}$} & \multirow{2}{*}{ AIC } \\
\hline & & & Wald & g.l. & p-valor & & \\
\hline Intercepto & 7,7666 & 0,3047 & 649,7 & 1 & $<0,001$ & \multirow{2}{*}{0,210} & \multirow{2}{*}{6170} \\
\hline IDHM & $-13,0378$ & 0,4731 & 759,5 & 1 & $<0,001$ & & \\
\hline Intercepto & $-3,4864$ & 0,1753 & 395,6 & 1 & $<0,001$ & \multirow{2}{*}{0,071} & \multirow{2}{*}{6789} \\
\hline Ln (F_PBF) & 0,4000 & 0,0245 & 267,6 & 1 & $<0,001$ & & \\
\hline Intercepto & $-16,265$ & 0,0616 & 698,0 & 1 & $<0,001$ & \multirow{2}{*}{0,083} & \multirow{2}{*}{6740} \\
\hline TX_RURAL & 0,0245 & 0,0014 & 322,5 & 1 & $<0,001$ & & \\
\hline Intercepto & $-0,8378$ & 0,0343 & 596,3 & 1 & $<0,001$ & \multirow{2}{*}{0,015} & \multirow{2}{*}{7024} \\
\hline Ln (TXMIL_INDIGENA) & 0,2035 & 0,0266 & 58,5 & 1 & $<0,001$ & & \\
\hline Intercepto & $-0,1433$ & 0,0529 & 7,3441 & 1 & 0,007 & \multirow{2}{*}{0,051} & \multirow{2}{*}{6876} \\
\hline (DENS_DEM) $1 / 2$ & $-0,0949$ & 0,0083 & 129,2 & 1 & $<0,001$ & & \\
\hline Intercepto & $-4,9364$ & 0,2073 & 566,9 & 1 & $<0,001$ & \multirow{2}{*}{0,131} & \multirow{2}{*}{6532} \\
\hline Ln (PO_AGRICULT_F) & 0,5842 & 0,0277 & 445,2 & 1 & $<0,001$ & & \\
\hline Intercepto & $-0,8426$ & 0,0313 & 723,6 & 1 & $<0,001$ & \multirow{4}{*}{0,039} & \multirow{4}{*}{6924} \\
\hline QUILOMO & - & - & - & - & - & & \\
\hline QUILOMBO = NÃO & . & . & . & . & . & & \\
\hline QUILOMBO = SIM & 1,0234 & 0,0811 & 159,2 & 1 & $<0,001$ & & \\
\hline Intercepto & 3,0601 & 0,1474 & 430,9 & 1 & $<0,001$ & \multirow{2}{*}{0,177} & \multirow{2}{*}{6323} \\
\hline IDEB_4 & $-0,8580$ & 0,0339 & 642,1 & 1 & $<0,001$ & & \\
\hline Intercepto & 2,7244 & 0,1623 & 281,9 & 1 & $<0,001$ & \multirow{2}{*}{0,119} & \multirow{2}{*}{6586} \\
\hline IDEB_8 & $-0,9217$ & 0,0438 & 443,1 & 1 & $<0,001$ & & \\
\hline Intercepto & $-0,9609$ & 0,0335 & 822,5 & 1 & $<0,001$ & \multirow{2}{*}{0,072} & \multirow{2}{*}{6788} \\
\hline Ln (NUM_FAM_ASS) & 0,2367 & 0,0140 & 286,2 & 1 & $<0,001$ & & \\
\hline Intercepto & $-1,0524$ & 0,0385 & 746,9 & 1 & $<0,001$ & \multirow{2}{*}{0,055} & \multirow{2}{*}{6859} \\
\hline Ln (PESCADORES) & 0,1998 & 0,0135 & 220,5 & 1 & $<0,001$ & & \\
\hline
\end{tabular}

Fonte: Elaborado pelos autores com base nos dados do Atlas do Desenvolvimento Humano (PNUD, 2013), MDS (2010), Censo Demográfico 2010 (IBGE) Censo Agropecuário 2006 (IBGE), Inep (2009), MDA (2010) e Fundação Cultural Palmares (2010).

Outra constatação importante que pode ser feita através da modelagem é avaliar qual das variáveis apresenta maior contribuição para a probabilidade de um município pertencer ao programa. Nesse sentido, ainda de acordo com a Tabela 2, os modelos que apresentaram maior Pseudo $\mathrm{R}^{2}$ e menor AIC (conjuntamente) foram, nesta ordem:

1o Índice de Desenvolvimento Humano (IDHM);

$2^{\mathrm{o}}$ Índice de Desenvolvimento da Educação Básica da rede pública, séries iniciais $\left(4^{\mathrm{a}}\right.$ série) (IDEB_4);

3o Pessoas ocupadas em agricultura familiar (pessoas de 14 anos e mais) (Ln (PO_AGRICULT_F));

$4^{\mathrm{o}}$ Índice de Desenvolvimento da Educação Básica da rede pública, séries finais $\left(8^{a}\right.$ série) (IDEB_8); 5o Percentual da população rural (TX_ RURAL);

6o Número de famílias assentadas (Ln (NUM_FAM_ASS));

7o Número de famílias beneficiárias do programa Bolsa Família (Ln (F_PBF));

$8^{\circ}$ Número de pescadores (pessoas de 10 anos ou mais) (Ln (PESCADORES));

9o Densidadedemográfica((DENS_DEM $\left.)^{1 / 2}\right)$;

$10^{\circ}$ Existência de Quilombos (QUILOMBO = SIM) e

11ํ População Indígena a cada mil habitantes (Ln (TXMIL_INDIGENA)).

Nesse sentido, pode-se afirmar que esta seja a ordem de prioridade dos critérios de elegibilidade. Observa-se que o IDH, índice sintético de qualidade de vida, largamente utilizado no monitoramento de políticas públicas, se apresenta 
como principal critério de elegibilidade, mostrando que o indicador se mantém relevante para o ciclo de monitoramento e avaliação de políticas públicas brasileiras. Além da qualidade de vida, a qualidade educacional se mostrou como relevante critério para a escolha de territórios da cidadania, mostrando o protagonismo e importância da educação básica no desenho do programa. A agricultura familiar, um dos principais pilares do PTC e de seus programas antecessores também se mostrou importante, mantendo a coerência do escopo e objetivos do programa no que tange à alocação de suas ações nas distintas regiões do País.

\section{Considerações finais}

O desenho diferenciado do PTC abordando a ação territorializada e a gestão social foi um processo evolutivo do Pronaf (criado em 1996) e do Pronat (criado em 2003). Os territórios da cidadania foram criados a partir da identificação de territórios rurais mais vulneráveis e da associação com critérios de elegibilidade e nenhum estudo de grande porte ajudou a corroborar a definição destes territórios e, consequentemente, a manutenção do programa, ou ainda com a definição dos critérios de elegibilidade. Nesse contexto, o presente trabalho apresenta uma metodologia estatística, utilizando dados secundários, que se mostrou adequada para avaliar e monitorar a totalidade de seus 120 territórios, possibilitando subsidiar decisões do Comitê Gestor do PTC.

O estudo empírico dos critérios de elegibilidade definidos no decreto de implantação apresentados neste trabalho teve a finalidade de compreender e evidenciar as associações entre os critérios e chance efetiva de um município pertencer ao programa. A utilização de dados secundários se mostrou relevante pelo custo, apesar do exaustivo trabalho de pesquisa, mostrando não ser necessário gerar informação primária para o diagnóstico do PTC. Além disso é importante ressaltar a preocupação de escolher fontes de dados com elevada credibilidade e confiabilidade.
A partir da análise descritiva, observou-se que, em geral, os critérios são atendidos, porém, analisando separadamente os critérios apresentados no $\S 1$ do artigo $1^{\mathrm{o}}$ (densidade populacional e volume populacional), foi possível observar que uma pequena parte dos municípios não eram elegíveis a serem atendidos pelo PTC. Através da modelagem estatística, que analisa tendências mais gerais, constatou-se que todos os critérios de elegibilidade possuem associação com a chance de um município pertencer ao programa, mas com certa variabilidade. Cabe ressaltar que a não associação com todos os critérios de elegibilidade, em algum município, não deve descartar a hipótese deste ser atendido pelo programa, visto que esta deve ser uma análise e uma decisão, considerando o conjunto de resultados apresentados.

A avaliação individual dos critérios de elegibilidade, realizada através dos modelos de regressão logística simples, possibilitou verificar o grau de importância de cada critério. Notou-se que critérios como densidade demográfica, existência de quilombos e população indígena; não apresentaram o protagonismo esperado. Estes critérios não estiveram no topo do ranqueamento por grau de importância. Este resultado não se mostrou aderente ao conteúdo do decreto de implantação do programa. O ranking encontrado foi: $1^{\mathrm{o}}$ Índice de Desenvolvimento Humano (IDHM); 2 o Índice de Desenvolvimento da Educação Básica da rede pública, séries iniciais (4⿳a série); 3 o Pessoas ocupadas em agricultura familiar (pessoas de 14 anos e mais); $4^{\mathrm{o}}$ Índice de Desenvolvimento da Educação Básica da rede pública, séries finais ( $8^{\underline{a}}$ série); $5^{\underline{o}}$ Percentual da população rural; $6^{0}$ Número de famílias assentadas; $7^{\circ}$ Número de famílias beneficiárias do programa Bolsa Família; 8o Número de pescadores (pessoas de 10 anos ou mais); 9o Densidade demográfica; $10^{\text {o }}$ Existência de Quilombos e 11o População Indígena a cada mil habitantes. A identificação do ranking foi importante para verificar, por exemplo, a prioridade dos critérios, conhecer melhor a elegibilidade dos territórios e vislumbrar com mais clareza as decisões do comitê gestor do programa. 
A partir da análise individual dos critérios de elegibilidade, desperta-se o interesse em avaliar a chance de o município ser atendido pelo PTC, a partir das Grandes Regiões do País e da análise conjunta dos critérios de elegibilidade. A avaliação conjunta, junto à avaliação do poder de discriminação dos critérios de elegibilidade, pode ser importante para identificação de novos territórios vulneráveis a serem atendidos pelo programa.

Deste modo, acredita-se que esse trabalho tenha fomentado um assunto pouco debatido no meio acadêmico e da gestão pública, o monitoramento do Programa Territórios da Cidadania, sobretudo na avaliação dos critérios de elegibilidade e estimação do grau de relevância de cada um deles. Espera-se, ainda, que a metodologia apresentada possa contribuir com as decisões do comitê gestor do PTC e de alguma forma ajudar nos próximos passos do programa.

\section{Referências}

ABRAMOVAY, R. Desenvolvimento sustentável: qual a estratégia para o Brasil? Novos Estudos - CEBRAP, São Paulo, n. 87, p. 97-113, jul. 2010.

BARBOSA, R. S., ZIMMERNANN, S. A. e TECCHIO, A. Desenvolvimento Territorial e pobreza rural no território da cidadania Serra Geral (MG). In: LEITE, S. P. Politicas de Desenvolvimento Territorial e Enfrentamento da Pobreza Rural no Brasil: estudos de casos. Brasília: ICCA, v. 20, p. 171-206, set. 2013.

BRASIL. Decreto de 24 de novembro de 2011. Altera o Decreto de 25 de fevereiro de 2008, que institui o Programa Territórios da Cidadania. Diário Oficial [da] República Federativa do Brasil, Poder Executivo, Brasília, DF, 25 nov. 2011. Seção 1, p. 3.

BRASIL. Decreto de 25 de fevereiro de 2008. Institui o Programa Territórios da Cidadania e dá outras providências. Diário Oficial [da] República Federativa do Brasil, Poder Executivo, Brasília, DF, 26 fev. 2008. Seção 1, p. 1.

BRASIL. Decreto de 23 de março de 2009. Dá nova redação aos arts. $1^{\circ}, 3^{-}$e $6^{0}$ do Decreto de 25 de fevereiro de 2008, que institui o Programa Territórios da Cidadania, e dá outras providências. Diário Oficial [da] República Federativa do Brasil, Poder Executivo, Brasília, DF, 24 mar. 2009. Seção 1, p. 1-13.
CAVALCANTI, M. M. A. Avaliação de políticas públicas e programas governamentais: uma abordagem conceitual. 13 p. Disponível em: < http://www.socialiris.org/antigo/ imagem/boletim/arq48975df171def.pdf $>$. Acesso em: 15 mar. 2015.

DELGADO, N. G. e LEITE, S. P. Políticas de Desenvolvimento Territorial no Meio Rural Brasileiro: Novas Institucionalidade e Protagonismo dos Atores. DADOS - Revista de Ciências Sociais, Rio de Janeiro, v. 54, n. 2, p. 431-473, 2011.

DEMARIS, A. Logit modeling: practical applications. Newbury Park: SAGE, serie 07-086, 1992, 91 p.

DOBSON, A. J. e BARNETT, A. G. An introdution to generalized linear models. London; Chapman and Hall/ CRC, 3. ed., 2002, 320 p.

FAVARETO, A. A abordagem territorial do desenvolvimento rural - mudança institucional ou "inovação por adição"? Estudos Avançados, São Paulo, v. 24 , n. 68 , p. 299-319, 2010.

HARRELL, F. E. rms: Regression Modeling Strategies. $R$ package, version 4.1-0, 2013, $241 \mathrm{p}$.

LASA, C. e DELGADO, N. G. Desenvolvimento Territorial e Enfrentamento da pobreza rural no território de Irecê (BA). In: LEITE, S. P. Políticas de Desenvolvimento Territorial e Enfrentamento da Pobreza Rural no Brasil: estudos de casos. Brasília: ICCA, v. 20, p. 75-138, set. 2013.

LOPES, E. S., DELGADO, N. G. e GRISA, C. Desenvolvimento territorial, políticas públicas e enfrentamento da pobreza no território Sertão Ocidental (SE). In: LEITE, S. P. Políticas de Desenvolvimento Territorial e Enfrentamento da Pobreza Rural no Brasil: estudos de casos. Brasília: ICCA, v. 20, p. 139-170, set. 2013.

LOPES, J. F. R. Território e participação política: o colegiado territorial Norte fluminense. 2012. 120 f. Dissertação (Mestrado em Administração Pública) - Escola Brasileira de Administração Pública e de Empresas da Fundação Getulio Vargas (EBAPE/FGV), Rio de janeiro, 2012.

MARSHALL, R. J. Mapping disease and mortality rates using Empirical Bayes Estimators. Journal of the Royal Statistical Society, Series C (Applied Statistics), London, v. 40, n. 2, p. 283-294, 1991.

MELO, W. S. Quando o passado se faz presente: os desafios da gestão social no programa de agricultura familiar. 2011. 158 f. Dissertação (Mestrado em Administração Pública) - Escola Brasileira de Administração Pública e de Empresas da Fundação Getulio Vargas (EBAPE/ FGV), Rio de janeiro, 2011. 
MELO, W. S., ANRADE, P. G. e AZEM, P. A participação como variável: a importância dos conselhos na atual política de desenvolvimento territorial no Brasil. In: CONGRESSO DE CIÊNCIAS SOCIAIS E HUMANAS EM LÍNGUA PORTUGUESA, 12., 2015, Portugal/ Lisboa, Anais... Portugal: UNL, 2015.

OLIVEIRA, J. R. et al. A Gestão Social no Contexto do Programa Territórios da Cidadania: os casos dos Municípios de Braga, Campo Novo e Coronel Bicaco - RS. Administração Pública e Gestão Social, Viçosa, v. 3, n. 1, p. 43-65, 2011.

PEREIRA, J. R. et al. Gestão Social dos Territórios da Cidadania: o zoneamento econômico-ecológico como instrumento de gestão do território noroeste de Minas Gerais. Cadernos EBAPE.BR, Rio de Janeiro, v. 9, n. 3, p. 724-747, set. 2011.

R CORE TEAM. foreign: Read Data Stored by Minitab, S, SAS, SPSS, Stata, Systat, Weka, dBase, .... R package, version 0.8-57, 2013.

ROSAR, D. B., CAZELLA, A. e ZIMMERMANN, S. A. Políticas de desenvolvimento territorial e enfrentamento da pobreza rural no território indígena Raposa Serra do Sil e São Marcos. In: LEITE, S. P. Politicas de Desenvolvimento Territorial e Enfrentamento da Pobreza Rural no Brasil: estudos de casos. Brasília: ICCA, v. 20, p. 207-248, set. 2013.

RÜCKERT, A. A. A Política Nacional de Ordenação Territorial, Brasil: uma política territorial contemporânea em construção. Scripta Nova - Revista Electrónica de Geografía y Ciencias Sociales, Barcelona, v. 11 , n. 245 (66), ago. 2007, 17 p.
SANTOS, R. A. Desafios do desenvolvimento territorial para agricultura familiar. In: SAQUET, M. A., SUZUKI, J. C. e MARAFON, G. J. (Org.). Territorialidades $e$ diversidade nos campos e nas cidades latino-americanas e francesas. São Paulo: Outras Expressões, v. 1, p. 321-334, 2011.

TECCHIO, A. e CAZELLA, A. A. Políticas de desenvolvimento territorial e superação da pobreza rural no Território Meio Oeste Contestado. In: LEITE, S. P. Politicas de Desenvolvimento Territorial e Enfrentamento da Pobreza Rural no Brasil: estudos de casos. Brasília: ICCA, v. 20, p. 37-74, set. 2013.

UNIVERSITY OF CALIFORNIA. What are pseudo R-squareds? 2011. Disponível em: < http://www.ats.ucla. edu/stat/mult_pkg/faq/general/Psuedo_RSquareds. htm > . Acesso em: 10 jul. 2014.

WARNES, G. R. et al. gmodels: Various R programming tools for model fitting. $\mathrm{R}$ package, version 2.15.4.1, 2013.

ZANI, F. B. Gestão social do desenvolvimento: a exclusão dos representantes dos empresários?: o caso do Programa Territórios da Cidadania Norte-RJ. 2010. 172 f. Dissertação (Mestrado em Administração Pública) - Escola Brasileira de Administração Pública e de Empresas da Fundação Getulio Vargas (EBAPE/FGV), Rio de janeiro, 2010.

ZIMMERMANN, S. A. et al. Desenvolvimento territorial e políticas de enfrentamento da pobreza rural no Brasil. Campo-Território: Revista de geografia agrária, v. 9, n. 17, p. 540-573, abr. 2014. 